\title{
Video Article \\ Experimental Protocol for Examining Behavioral Response Profiles in Larval Fish: Application to the Neuro-stimulant Caffeine
}

\author{
W. Baylor Steele ${ }^{1,2}$, Rachel A. Mole ${ }^{1}$, Bryan W. Brooks ${ }^{1,2}$ \\ ${ }^{1}$ Department of Environmental Science, Center for Reservoir and Aquatic Systems Research, Baylor University \\ ${ }^{2}$ Institute of Biomedical Studies, Baylor University
}

Correspondence to: Bryan W. Brooks at Bryan_Brooks@baylor.edu

URL: https://www.jove.com/video/57938

DOI: doi: $10.3791 / 57938$

Keywords: Environmental Sciences, Issue 137, Behavioral Response Profiling, Behavioral Toxicology, Zebrafish, Fathead Minnow, Caffeine, Neuro Stimulant

Date Published: 7/24/2018

Citation: Steele, W.B., Mole, R.A., Brooks, B.W. Experimental Protocol for Examining Behavioral Response Profiles in Larval Fish: Application to the Neuro-stimulant Caffeine. J. Vis. Exp. (137), e57938, doi:10.3791/57938 (2018).

\section{Abstract}

Fish models and behaviors are increasingly used in the biomedical sciences; however, fish have long been the subject of ecological, physiological and toxicological studies. Using automated digital tracking platforms, recent efforts in neuropharmacology are leveraging larval fish locomotor behaviors to identify potential therapeutic targets for novel small molecules. Similar to these efforts, research in the environmental sciences and comparative pharmacology and toxicology is examining various behaviors of fish models as diagnostic tools in tiered evaluation of contaminants and real-time monitoring of surface waters for contaminant threats. Whereas the zebrafish is a popular larval fish model in the biomedical sciences, the fathead minnow is a common larval fish model in ecotoxicology. Unfortunately, fathead minnow larvae have received considerably less attention in behavioral studies. Here, we develop and demonstrate a behavioral profile protocol using caffeine as a model neurostimulant. Though photomotor responses of fathead minnows were occasionally affected by caffeine, zebrafish were markedly more sensitive for photomotor and locomotor endpoints, which responded at environmentally relevant levels. Future studies are needed to understand comparative behavioral sensitivity differences among fish with age and time of day, and to determine whether similar behavioral effects would occur in nature and be indicative of adverse outcomes at the individual or population levels of biological organization.

\section{Video Link}

The video component of this article can be found at https://www.jove.com/video/57938/

\section{Introduction}

Though fish models are increasingly used for biomedical studies, fish have been routinely employed for ecology and physiology studies, to examine contamination of surface waters, and to understand toxicological thresholds of chemicals. Such efforts are important because chemical contamination can impair aquatic ecosystems and jeopardize the quality of source water supplies ${ }^{1,2}$. Most of the chemicals in commerce, however, lack even basic toxicology information ${ }^{3}$.

Animal model assays traditionally used in regulatory toxicity testing are resource intensive and cannot provide the high throughput, early tier screening needed for toxicity testing in the 21 st century ${ }^{4}$. Subsequently, there is a growing impetus to adopt and utilize in vitro models that can more rapidly and efficiently screen compounds for biological activities ${ }^{3,5}$. Though cell based models present many opportunities, they often lack biological complexity, and thus do not account for many important whole organism processes, including metabolism .

The zebrafish is a common biomedical animal model that is gaining popularity as an alternative model in aquatic toxicology and ecotoxicology $y^{7,8}$ Given their small size, rapid development, and high fecundity, fish models can be used to rapidly and efficiently screen chemicals for bioactivity and toxicity at the whole organism scale ${ }^{9}$. With the aid of automated tracking software, larval zebrafish behaviors provide enhanced diagnostic utility in screening contaminants for toxicity ${ }^{10,11}$. Studies in the pharmaceutical sciences have demonstrated that locomotor endpoints are informative of chemical mechanisms of action, can be used to phenotype behaviors, and then may tentatively identify subcellular targets for novel molecules ${ }^{12,13}$. Whereas the zebrafish is a popular larval fish model in the biomedical sciences, the fathead minnow is a common, ecologically important fish model that is used for ecotoxicology studies and during prospective (e.g., new chemical evaluations) and retrospective (e.g., ambient surface water or wastewater effluent discharge monitoring) environmental assessments. Unfortunately, behavioral responses of larval fathead minnows have received markedly less attention than zebrafish. Our ongoing research with two common larval fish models, the zebrafish and fathead minnow, suggests that larval fish swimming patterns appear unique to anticipated modes or mechanisms of action for diverse chemicals. Thus, behavioral endpoints provide the potential to rapidly and sensitively examine chemicals for toxicity and to identify subcellular targets for industrial chemical and other contaminants, particularly during early tier assessments.

Here, we report a protocol for examining behavioral response profiles in larval fish. We demonstrate these methods using caffeine, a model neurostimulant and a common aquatic contaminant that is introduced to aquatic systems through discharge from wastewater treatments plants following human consumption of foods, beverages, and pharmaceuticals formulated with caffeine ${ }^{14}$. We examine behavioral responses 
to caffeine in both larval zebrafish and fathead minnow, including to a sudden change in lighting condition, which is often referred to as a photomotor response (PMR) during pharmaceutical studies with embryonic and larval zebrafish ${ }^{13,15}$. We further identify effects of caffeine across several locomotor endpoints to develop chemical response profiles for each fish model. Caffeine treatment levels used in this study represent the upper centiles of exposure distributions based on measured environmental values of caffeine ${ }^{16}$. We also include treatments benchmarked to larval fish $\mathrm{LC}_{50}$ values, and the therapeutic hazard value (THV), a pharmaceutical concentration in water that is anticipated to result in plasma levels in fish consistent with a human therapeutic plasma dose.

\section{Protocol}

Studies in this protocol generally follow standardized experimental design and recommended statistical analysis guidelines from the US Environmental Protection Agency (EPA no. 2000.0) for fathead minnows and the Organization for Economic Cooperation and Development (OECD no. 236) for zebrafish. These experimental designs (e.g., increasing replication) can be modified within the current protocol for future studies. Fish culture conditions follow previously published literature ${ }^{17}$. All experimental procedures and fish culture protocols followed Institutional Animal Care and Use Committee protocols approved at Baylor University.

\section{Exposing Fish to Chemical Treatment}

1. Prepare caffeine exposure solutions by dissolving caffeine in reconstituted hard water. Perform appropriate serial dilutions by diluting higher caffeine treatments with hard water to produce lower caffeine treatment levels.

NOTE: Table 1 summarizes each of the treatment levels used in this experiment.

\begin{tabular}{|l|l|l|l|l|l|}
\hline & Zebrafish & & \multicolumn{2}{l|}{ Fathead Minnow } \\
\hline Treatment & $\begin{array}{l}\text { Nominal Caffeine } \\
\text { Concentration (mg/L) }\end{array}$ & $\begin{array}{l}\text { Measured Caffeine } \\
\text { Concentration (mg/L) }\end{array}$ & Treatment & $\begin{array}{l}\text { Nominal Caffeine } \\
\text { Concentration (mg/L) }\end{array}$ & $\begin{array}{l}\text { Measured Caffeine } \\
\text { Concentration (mg/L) }\end{array}$ \\
\hline Control & 0 & LOD & Control & 0 & $<$ LOD \\
\hline 75th Centile* & 0.001 & 0.001 & 75 th Centile* & 0.001 & 0.001 \\
\hline 95 th Centile* & 0.039 & 0.013 & 95 th Centile* & 0.039 & 0.009 \\
\hline 99 th Centile* & 0.412 & 0.361 & 99 th Centile* & 0.412 & 0.310 \\
\hline THV & 4.07 & 3.81 & THV & 4.07 & 4.12 \\
\hline $10 \%$ LC50 & 48.46 & 46.66 & $10 \%$ LC50 & 14.1 & 14.7 \\
\hline $40 \%$ LC50 & 193.82 & 186.67 & $40 \%$ LC50 & 56.38 & 53.91 \\
\hline
\end{tabular}

Table 1: Experimental caffeine treatments for zebrafish and fathead minnow experiments. Nominal and measured values of caffeine for each treatment are given. *The caffeine treatments used in this study represent the upper centiles of exposure distributions based on measured environmental values of caffeine ${ }^{16}$. THV: Therapeutic Hazard Value. LOD: Limit of Detection

2. Pour the prepared solution in individual exposure champers. Use $100 \mathrm{~mL}$ glass beakers filled with $20 \mathrm{~mL}$ of exposure solution for zebrafish exposure chambers and $500 \mathrm{~mL}$ beakers with $200 \mathrm{~mL}$ of exposure solution for fathead minnow exposure chambers.

3. Using a transfer pipette, place 10 zebrafish embryos aged $4-6 \mathrm{~h}$ post fertilization (hpf) in each of four replicate exposure chambers per treatment.

4. Place 10 fathead minnow larvae aged within $24 \mathrm{~h}$ of hatching in each of three replicate exposure chambers per treatment. To accommodate the larger size of fathead minnow larvae, cut the tip of the transfer pipette off prior to transfer.

5. Maintain zebrafish experiments at a $16: 8 \mathrm{~h}$ light:dark photoperiod and a constant temperature of $28 \pm 1{ }^{\circ} \mathrm{C}$. Use the same photoperiod regime for fathead minnow studies, but at a temperature of $25 \pm 1{ }^{\circ} \mathrm{C}$.

6. After $\mathbf{9 6} \mathrm{h}$ of chemical exposure, load individual fish in separate wells of $\mathbf{4 8}$ (for zebrafish) and $\mathbf{2 4}$ (for fathead minnow) well plates.

1. To ensure that each well contains an equal volume of solution, transfer zebrafish larvae to 48 well plates using a $5,000 \mu \mathrm{L}$ autopipette for a $1,000 \mu \mathrm{L}$ volume per well. Use the autopipette to withdraw and transfer both the zebrafish larvae and exposure solution simultaneously.

2. Due to their larger size, transfer fathead minnow larvae using a transfer pipette with the tip cut off. Prior to transferring fathead minnow larvae to individual wells, fill each well to $2,000 \mu \mathrm{L}$ using an autopipette. When transferring individual fathead larvae to wells, place the tip of the transfer pipette in the well solution and allow the fish to swim from the pipette tip into the well.

\section{Calibration of Video-tracking Parameters}

1. Prior to behavioral measures, set observation and calibration parameters in the video track software (see Table of Materials).

1. Place a well plate in the recording chamber with at least 1 larval fish in an individual well. Use the plate and associated fish as representations to set calibration parameters.

2. In the video track software, click "File | Generate Protocol", which will open a "protocol creation wizard" dialogue box. In the "Location Count" field, enter the number of individuals wells of the well plate and then click "OK".

3. At the top of the screen, click "View | Full Screen", which will prompt the system to display an overhead camera view of the well plate.

4. Click the "Draw Areas" icon, which appears as three multicolored shapes. To the right of the well plate viewing area, select the circle icon in the field labeled "Areas". 
5. Use the cursor to delineate the circular video tracking area in the top left well of the well plate. Select "Top-Right Mark" and then outline the viewing area of the top right well. Then, select "Bottom Mark" to outline the bottom right well.

NOTE: After drawing the circular outline, its position will likely need to be adjusted. To adjust the position of the outline, click "select" and then use the cursor to move the outlined area. Also, outlines can be replicated by clicking "Copy" and then "Paste".

6. After the top left, top right, and bottom right well tracking areas have been defined, click "Build" to prompt the software to automatically delineate the viewing areas of the remaining wells.

7. In the area labeled "Calibration", click "Draw Scale". Use the cursor to draw a horizontal line across the plate. Once the line is drawn, a dialogue box labeled "Calibration measurement" will appear. Enter the well plate length and click "OK".

8. Exit the drawing manager by clicking the "Draw Areas" icon.

9. Click the "Tiles" icon. Using the cursor, highlight all the boxes that appear on the viewing screen so that each box is green. NOTE: The Tiles icon appears as a group of six individual small squares

10. "Click View| Full Screen". To the right of the plate viewing area, click "Bkg" in the box labeled "Detection Threshold". Use the threshold adjustment bar to set the pixel detection threshold. Once, the appropriate pixel detection threshold is selected, click "Apply to Group". NOTE: This protocol sets the detection threshold at 13 in black mode for zebrafish observations and at 110 in transparent mode for fathead minnow observations.

11. In the box labeled "Movement Threshold", enter the desired movement speed tracking parameters. Once speed parameters are set, click "Apply to Group".

NOTE: This protocol sets small/large movements at $20 \mathrm{~mm} / \mathrm{s}$ and inactive/small movements at $5 \mathrm{~mm} / \mathrm{s}$. These selections program the software to track larval fish movement at three different speed levels: inactive (freezing) $=<5 \mathrm{~mm} / \mathrm{s}$, small (cruising) $=5-20 \mathrm{~mm} / \mathrm{s}$, and large (bursting) $=>20 \mathrm{~mm} / \mathrm{s}$.

12. Click "Parameters | Protocol Parameters" from the drop-down menu. In the dialogue box, select the "Time" tab. Enter the observation time and the integration time. After parameters are entered click "Ok".

13. To set the light/dark photoperiod times and light intensity for each photoperiod open the light driver settings dialogue box by selecting "Light Driving" from the "Parameters" drop down menu.

NOTE: See protocol video for setting multiple light-dark photoperiods.

14. After the video tracking parameters have been set, save the observation protocol.

NOTE: This protocol observes fish behavior over a 50 min period that includes a 10 min acclimation phase followed by 4 altering light/ dark phases consisting of two $10 \mathrm{~min}$ light periods and two $10 \mathrm{~min}$ dark periods. The integration time is set to measure behavior for each minute of the 50 min behavioral trial.

\section{Observation of Larval Fish Locomotor and Photomotor Behavior}

1. Place the well plate containing experimental fish in the behavioral recording chamber.

2. In the video tracking software, open the tracking protocol developed in step 3.

3. In the video tracking viewer, check to make sure that all larvae are visible on the computer screen, that only one individual larva is present in each well, and that individual wells are aligned within the observation areas that were defined in steps 2.1.5 and 2.1.6.

4. Click on "Experiment | Execute". NOTE: The system will prompt the user to provide a name and location to save the observation data.

5. Once the name and save location of the observation data have been specified, click on the "Several Live Images" icon to highlight all the predefined viewing areas

NOTE: This icon is located at the top of the computer screen and appears as a box divided into four smaller squares. Clicking on this icon will highlight all the pre-defined viewing areas.

6. Close the panel of the recording chamber and click "Background | Start" on the computer monitor.

\section{Analyzing Behavioral Data}

1. To retrieve larval fish activity data, open the spreadsheet, which is automatically compiled by the tracking software and is in the folder specified by the user before initiating behavioral trials (Step 3.4).

2. Refer to Figures $\mathbf{A}$ and $\mathbf{1 B}$ for representative measurements of naive locomotor activity of unexposed zebrafish and fathead minnow larvae, respectively. Refer to Figures $1 \mathrm{C}$ and 1D for PMR calculations, which effectively examine the magnitude of movement difference between light to dark or dark to light transitions. 

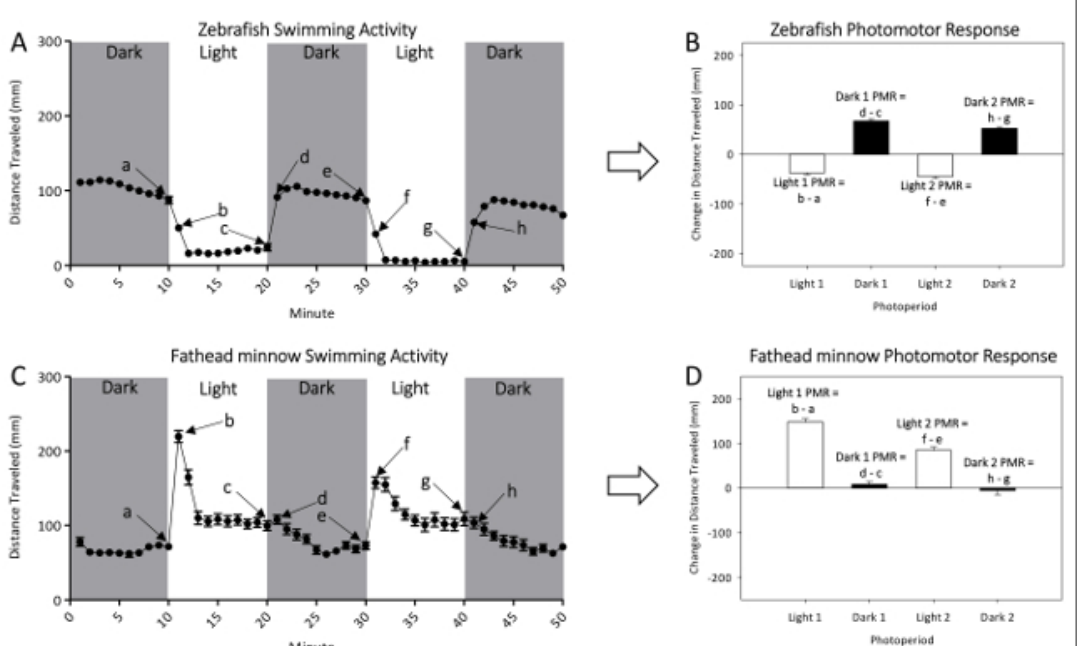

Figure 1: Example of baseline activity of unexposed zebrafish (A and $\mathbf{B})$ and fathead minnow (C and $\mathbf{D})$. The mean $( \pm$ SEM) distance swam for zebrafish (A) and fathead minnow (C) is given by dots each representing one-minute intervals of activity. Two dark and two light periods of photomotor responses are measured. The last ( $a, c, e$, and $g$ ) and first (b, d, f, and h) minute of each photoperiod are used to calculate PMRs. Photomotor responses of zebrafish (B) and fathead minnow (D) are measured as the change in mean ( \pm SEM) distance traveled between the last minute of an initial photoperiod and the first minute of the following period. Please click here to view a larger version of this figure.

\section{Representative Results}

Caffeine treatment levels did not appreciably vary during the $96 \mathrm{~h}$ experiments with zebrafish and fathead minnows. For example, Table 1 presents analytically verified concentrations of each treatment level. This protocol verified water samples for caffeine treatment levels by isotopedilution liquid chromatography tandem mass spectrometry (LC-MS/MS) generally following previously reported methods ${ }^{28}$. The formation of paraxanthine, the primary metabolite of caffeine, was also quantified. A description of these analytical procedures is provided in the supplemental analytical information. Because of the similarities between nominal and analytical verification of treatments, nominal treatment levels are presented throughout the remainder of this manuscript. Caffeine significantly altered zebrafish and fathead minnow behaviors. However, zebrafish locomotor responses were consistently more sensitive to caffeine than fathead minnows. The most sensitive behavioral endpoints for zebrafish and fathead minnow larvae were affected by caffeine at a concentration of $0.039 \mathrm{mg} / \mathrm{L}$. Table 2 summarizes lowest observed effect concentrations (LOECs) and no observed effect concentrations (NOECs) for each behavioral endpoint in both fish models. 


\begin{tabular}{|c|c|c|c|c|c|}
\hline \multicolumn{3}{|l|}{ Zebrafish } & \multicolumn{3}{|l|}{ Fathead minnow } \\
\hline Endpoint & LOEC (mg/L) & NOEC (mg/L) & Endpoint & LOEC (mg/L) & NOEC (mg/L) \\
\hline Total Distance Dark & 0.412 & 0.039 & Total Distance Dark & - & 56.38 \\
\hline Total Distance Light & 48.46 & 4.07 & Total Distance Light & - & 56.38 \\
\hline Total Counts Dark & 0.412 & 0.039 & Total Counts Dark & - & 56.38 \\
\hline Total Counts Light & 48.46 & 4.07 & Total Counts Light & - & 56.38 \\
\hline Bursting Distance Dark & - & 193.82 & Bursting Distance Dark & - & 56.38 \\
\hline $\begin{array}{l}\text { Bursting Distance } \\
\text { Light }\end{array}$ & 193.82 & 48.46 & $\begin{array}{l}\text { Bursting Distance } \\
\text { Light }\end{array}$ & - & 56.38 \\
\hline Bursting Counts Dark & 193.82 & 48.46 & Bursting Counts Dark & - & 56.38 \\
\hline Bursting Counts Light & 193.82 & 48.46 & Bursting Counts Light & - & 56.38 \\
\hline Bursting Duration Dark & 193.82 & 48.46 & Bursting Duration Dark & - & 56.38 \\
\hline Bursting Duration Light & - & 193.82 & Bursting Duration Light & - & 56.38 \\
\hline Cruising Distance Dark & 0.412 & 0.039 & Cruising Distance Dark & - & 56.38 \\
\hline $\begin{array}{l}\text { Cruising Distance } \\
\text { Light }\end{array}$ & 48.46 & 4.07 & $\begin{array}{l}\text { Cruising Distance } \\
\text { Light }\end{array}$ & - & 56.38 \\
\hline Cruising Counts Dark & 0.412 & 0.039 & Cruising Counts Dark & - & 56.38 \\
\hline Cruising Counts Light & 48.46 & 4.07 & Cruising Counts Light & - & 56.38 \\
\hline Cruising Duration Dark & 0.412 & 0.039 & Cruising Duration Dark & - & 56.38 \\
\hline Cruising Duration Light & 48.46 & 4.07 & Cruising Duration Light & - & 56.38 \\
\hline $\begin{array}{l}\text { Freezing Distance } \\
\text { Dark }\end{array}$ & 0.412 & 0.039 & $\begin{array}{l}\text { Freezing Distance } \\
\text { Dark }\end{array}$ & 0.039 & 0.001 \\
\hline $\begin{array}{l}\text { Freezing Distance } \\
\text { Light }\end{array}$ & 0.039 & 0.001 & $\begin{array}{l}\text { Freezing Distance } \\
\text { Light }\end{array}$ & - & 56.38 \\
\hline Freezing Counts Dark & 0.412 & 0.039 & Freezing Counts Dark & - & 56.38 \\
\hline Freezing Counts Light & 48.46 & 4.07 & Freezing Counts Light & - & 56.38 \\
\hline $\begin{array}{l}\text { Freezing Duration } \\
\text { Dark }\end{array}$ & - & 193.82 & $\begin{array}{l}\text { Freezing Duration } \\
\text { Dark }\end{array}$ & 56.38 & 14.10 \\
\hline $\begin{array}{l}\text { Freezing Duration } \\
\text { Light }\end{array}$ & 48.46 & 4.07 & $\begin{array}{l}\text { Freezing Duration } \\
\text { Light }\end{array}$ & - & 56.38 \\
\hline Dark 1 PMR & 48.46 & 4.07 & Dark 1 PMR & 0.039 & 0.001 \\
\hline Light 1 PMR & 48.46 & 4.07 & Light 1 PMR & - & 56.38 \\
\hline Dark 2 PMR & 48.46 & 4.07 & Dark 2 PMR & - & 56.38 \\
\hline Light 2 PMR & 48.46 & 4.07 & Light 2 PMR & - & 56.38 \\
\hline Bursting Dark 1 PMR & - & 193.82 & Bursting Dark 1 PMR & - & 56.38 \\
\hline Bursting Light 1 PMR & - & 193.82 & Bursting Light 1 PMR & - & 56.38 \\
\hline Bursting Dark 2 PMR & 193.82 & 48.46 & Bursting Dark 2 PMR & - & 56.38 \\
\hline Bursting Light 2 PMR & - & 193.82 & Bursting Light 2 PMR & - & 56.38 \\
\hline Cruising Dark 1 PMR & 48.46 & 4.07 & Cruising Dark 1 PMR & - & 56.38 \\
\hline Cruising Light 1 PMR & 48.46 & 4.07 & Cruising Light 1 PMR & - & 56.38 \\
\hline Cruising Dark 2 PMR & 48.46 & 4.07 & Cruising Dark 2 PMR & - & 56.38 \\
\hline Cruising Light 2 PMR & 193.82 & 48.46 & Cruising Light 2 PMR & 56.38 & 14.10 \\
\hline Freezing Dark 1 PMR & 48.46 & 4.07 & Freezing Dark 1 PMR & - & 56.38 \\
\hline Freezing Light 1 PMR & 193.82 & 48.46 & Freezing Light 1 PMR & - & 56.38 \\
\hline Freezing Dark 2 PMR & 48.46 & 4.07 & Freezing Dark 2 PMR & - & 56.38 \\
\hline Freezing Light 2 PMR & 193.82 & 48.46 & Freezing Light 2 PMR & - & 56.38 \\
\hline
\end{tabular}

Table 2: Zebrafish and fathead minnow behavioral NOECs and LOECs for Caffeine. No Observed Effect Concentration (NOEC) and Lowest Observed Effect Concentration (LOEC) (mg/L) values for each of the light/dark swimming activity endpoints and photomotor responses for 
zebrafish and fathead minnows exposed to caffeine. Dashes indicate that no effects were observed at a particular endpoint across all treatment levels.

Figure 2 presents total locomotor activity and PMRs of zebrafish and fathead minnow following $96 \mathrm{~h}$ exposure to caffeine. Fathead minnow larvae PMRs were altered by caffeine at lower treatment levels $(0.038 \mathrm{mg} / \mathrm{L})$ than zebrafish, but a markedly larger number of photomotor endpoints were affected in zebrafish. The highest treatment level of caffeine $(193.82 \mathrm{mg} / \mathrm{L})$ altered PMR in zebrafish, in which these responses were exactly opposite from controls. At this elevated treatment level, however, PMRs decreased in dark and increased in light conditions.
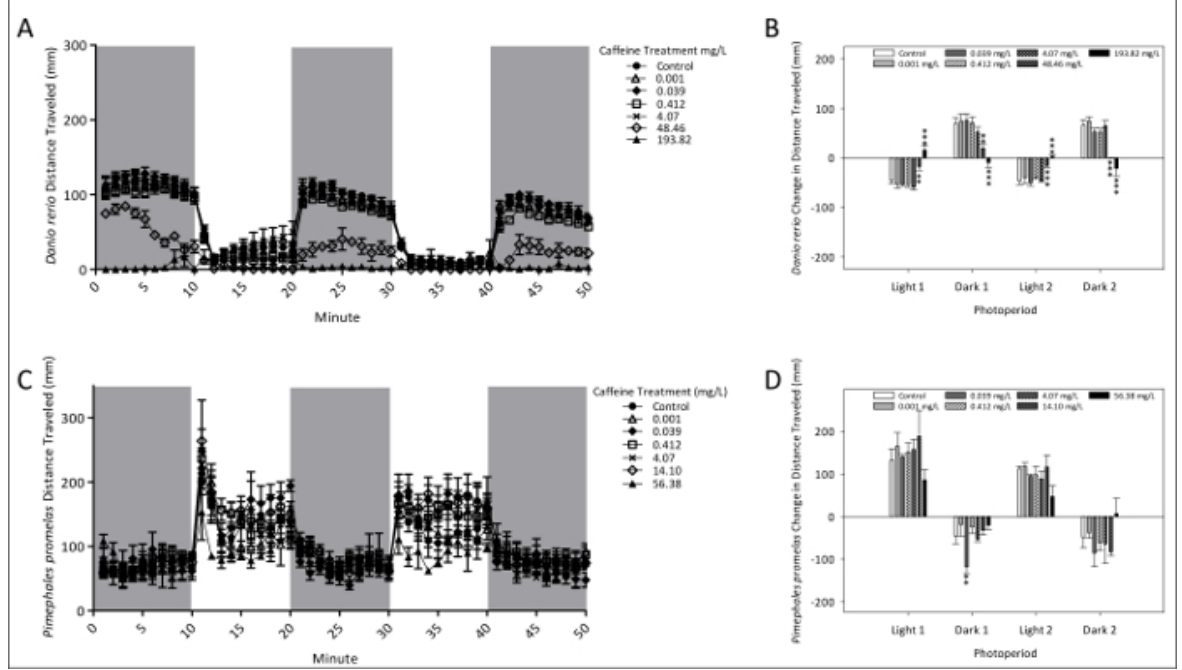

Figure 2: Swimming activity and photomotor responses of zebrafish (A and $\mathbf{B})$ and fathead minnow (C and $\mathbf{D})$ after $96 \mathrm{~h}$ exposure to caffeine. The mean $( \pm$ SEM) distance swam for zebrafish $(\mathbf{A})$ and fathead minnow $(\mathbf{C})$ is given by dots each representing 1-min intervals of activity. Photomotor responses of zebrafish (B) and fathead minnow (D) are measured as the change in mean $( \pm$ SE) total distance traveled between the last minutes of an initial photoperiod and the first minute of the following period. Two dark and two light period photomotor responses were measured. A total of 24 (4 replicates each of 6 larvae) zebrafish and 12 ( 3 replicates each of 4 larvae) fathead minnows were used for behavioral observation. ${ }^{*} p<0.10 ;{ }^{* *} p<0.05 ;{ }^{* * *} p<0.01$. Please click here to view a larger version of this figure.

In addition to measuring larval PMRs, light and dark locomotor activity was analyzed across three speed thresholds for distance moved, number of movements, and duration of movements. This data is used to develop behavioral response profiles for caffeine (Figure 3, Supplemental Figure 1). In both of the fish models, caffeine inhibited activity at all significantly affected locomotor endpoints. Both fish models demonstrated increased activity at the bursting speed thresholds following exposure to caffeine, though not significantly. Similar to the results of the PMR observations, caffeine effected a greater number of zebrafish locomotor endpoints. In fact, caffeine significantly altered several locomotor responses under dark conditions at environmentally realistic levels below the THV. However, fathead minnow locomotor activity was not significantly affected under light conditions by any treatment level. 

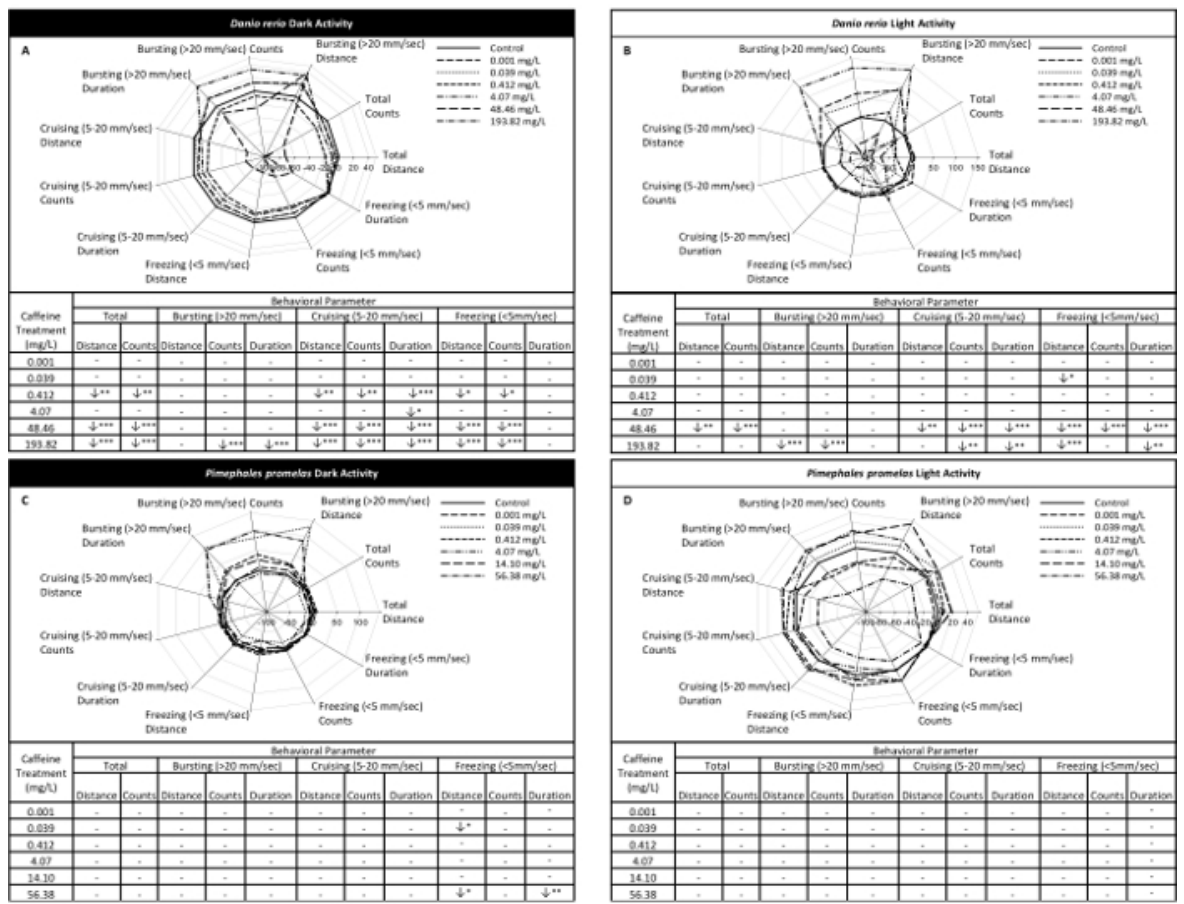

Figure 3: Response profiles of larval zebrafish and fathead minnows after $96 \mathrm{~h}$ exposure to caffeine. Mean zebrafish dark (A) and light (B) swimming activity compared to mean fathead minnow dark (C) and light (D) activity after $96 \mathrm{~h}$ exposure to caffeine. Plotted data represents activity over two 10 min dark photoperiods and two $10 \mathrm{~min}$ light photoperiods for each fish model. Data is normalized to control, which is represented at the 0 axis in each figure. Behavioral parameters include distance swam, number of movements (counts), and duration of each movement across 3 speed levels, bursting $(>20 \mathrm{~mm} / \mathrm{s})$, cruising $(5-20 \mathrm{~mm} / \mathrm{s})$, and freezing $(<5 \mathrm{~mm} / \mathrm{s})$. In addition to movement patterns at each of the speed thresholds, total distance swam, and total number of movements is represented. $\uparrow$ represents a significant increase in activity in comparison to control and $\downarrow$ indicates a significant decrease in activity in comparison to control. A total of 24 (4 replicates each of 6 larvae) zebrafish and 12 (3 replicates each of 4 larvae) fathead minnow where used in behavioral observations for each group. ${ }^{*} p<0.10 ;{ }^{* *} p<0.05 ;{ }^{* * *} p$ $<0.01$. Please click here to view a larger version of this figure.

Supplemental Figure 1: Photomotor responses of zebrafish (A and B) and fathead minnow (C and $\mathbf{D})$ across three speed thresholds. Zebrafish $(\mathrm{A}, \mathrm{B}$, and $\mathrm{C})$ and fathead minnow larvae $(\mathrm{D}, \mathrm{E}$, and $\mathrm{F}$ ) photomotor responses across three speed thresholds (Freezing: $20 \mathrm{~mm} / \mathrm{s})$ after $96 \mathrm{hr}$ exposure to caffeine. Photomotor responses of zebrafish and fathead minnow are measured as the change in mean ( \pm SE) total distance traveled between the last minutes of an initial photoperiod and the first minute of the following period. Two dark and two light period photomotor responses were measured. A total of 24 ( 4 replicates each of 6 larvae) zebrafish and 12 ( 3 replicates of 4 larvae) fathead minnows were used for behavioral observation. ${ }^{*} p<0.01$ Please click here to download this file.

\section{Discussion}

When selecting chemical treatment levels for behavioral toxicology studies, several factors must be considered. Caffeine treatment levels in the present study were selected based on upper centile values for predicted environmental exposure scenarios from wastewater effluent ${ }^{16}$. When possible, we routinely select treatment levels for aquatic toxicology studies using probabilistic exposure assessments of environmental observations $^{19,20,21}$. A THV, which is calculable for medicines, was also included as a treatment level in the present study. THV values (Eq. 1$)^{22,23}$ are defined as predicted water concentrations leading to human therapeutic doses (Cmax) of pharmaceuticals in fish ${ }^{23}$, are inspired from initial plasma modeling efforts ${ }^{24}$, and are calculated based on blood:water chemical partitioning coefficients (Eq. 2$)^{25}$.

$\mathrm{THV}=\mathrm{C}_{\max } / \log \mathrm{P}_{\mathrm{BW}}$

$\log \mathrm{P}_{\mathrm{BW}}=\log \left[\left(10^{0.73 \cdot \log \mathrm{Kow}}\right.\right.$.

$0.16)+0.84]$

Here, we also select sublethal treatment levels relative to zebrafish and fathead minnow LC50 values. We consider this approach a useful benchmarking procedure for behavioral responses, particularly when comparing thresholds of specific behaviors with a fish model across multiple chemicals. It further facilitates calculations of acute to chronic ratios, which can be diagnostically useful in aquatic toxicology for mechanistic studies and assessments. LC50 values were obtained from preliminary toxicity bioassays following the standardized guidelines given in step 2.1.

In this protocol, we employ common experimental designs and statistical techniques recommended by the US EPA and OECD standardized methods for toxicology studies with fish models. Though we report $p$ values $(e . g .,<0.01,<0.05,<0.10)$, significant differences $(\alpha=0.10)$ in activity levels are identified among treatments using analysis of variance (ANOVA) if normality and equivalence of variance assumptions are met. Dunnett's or Tukey's HSD post hoc tests are performed to identify treatment level differences. We select this alpha $(\alpha=0.10)$ value to reduce type II errors, particularly for early tier assays and when an understanding of biologically important effect size is limited for understudied behavioral endpoints and model organisms ${ }^{26}$, instead of employing procedures more common in the biomedical sciences for 
multiple comparisons (e.g., Bonferroni correction for RNA-Seq data) ${ }^{27}$. Future studies are needed to understand variability of these behavioral responses and potentially modify experimental designs (e.g., increase replication) accordingly.

A number of factors can influence behavior of larval fish in addition to chemical exposure. For example, time of day, age, well size, temperature, lighting condition, and volume of exposure solution in each well represent important considerations ${ }^{11,30}$. For these reasons, precautions should be taken to minimize the effects of external factors that could influence locomotor behavior of the larval fish during experimentation. Behavioral observations should be performed in narrow time windows ( 3 to $4 \mathrm{~h}$ ) and across time periods when time of day effects are expected to have minimal influence on larval locomotor behavior ${ }^{11}$. Additionally, larval fish should be maintained at a consistent temperature $\left(28 \pm 1{ }^{\circ} \mathrm{C}\right.$ for zebrafish and $24 \pm 1{ }^{\circ} \mathrm{C}$ for $\mathrm{FHM}$ ) and on a defined light/dark cycle in temperature-controlled incubators throughout the exposure period. In addition, the temperature of the laboratory where behaviors are recorded should be maintained to conditions approximating experimental conditions to avoid temperature influences on behaviors. Further, wells used during behavioral observations should be maintained at a consistent volume for each individual fish.

Larval and embryonic zebrafish PMRs have been previously used in the biomedical sciences to identify potential therapeutic targets for novel compounds ${ }^{12,13}$. This protocol expands on previous behavioral research with zebrafish by utilizing 38 endpoints to investigate chemical bioactivity of environmental contaminants. Although caffeine is a common aquatic contaminant with an understood mechanism of action (MoA), many compounds in commerce lack important mechanistic data. Therefore, this protocol can be employed to gain insight of MoAs for compounds lacking toxicity data, including commercial chemicals ${ }^{39}$. Furthermore, the protocol provides methods for two of the most commonly used fish models. As noted previously, whereas the zebrafish is a common biomedical fish model that is becoming increasingly popular in ecotoxicology, the fathead minnow is commonly used as an ecological model for environmental assessment applications but has received comparatively less attention in behavioral studies with automated systems compared to the zebrafish. Though there remains no standardized regulatory methods for fish behavioral toxicology studies, this protocol provides an approach to support future efforts.

Caffeine elicited behavioral responses in each of the fish models at levels that have been detected in the aquatic environment ${ }^{16}$. RodriguezGil et al. 2018 developed global environmental exposure distributions in aquatic systems based on measured values of caffeine ${ }^{\text {i6 }}$. Specifically, $95 \%$ of predicted wastewater effluent concentrations would fall below the LOECs for the most sensitive behavioral endpoints of zebrafish and fathead minnow in the present study (Table 2). Though several behavioral effects of caffeine were observed in zebrafish (particularly in dark conditions) at environmentally relevant levels, it is unclear whether these behavioral modifications might occur in natural fish populations or result in ecologically important adverse outcomes. Though useful for sensitive, diagnostic screening purposes, larval fish behavioral thresholds may not be representative of other life history stages or of fish in natural populations. Further research is warranted to determine whether similar behavioral response thresholds would occur in nature and be indicative of adverse outcomes at the individual or population levels of biological organization.

\section{Disclosures}

The authors have nothing to disclose.

\section{Acknowledgements}

Support for this study was provided by the U.S. National Science Foundation (Project \#: CHE-1339637) with additional support from the U.S. Environmental Protection Agency. We thank Dr. Jone Corrales, Dr. Lauren Kristofco, Gavin Saari, Samuel Haddad, Bekah Burket and Bridgett Hill for general lab support.

\section{References}

1. Malaj, E., et al. Organic chemicals jeopardize the health of freshwater ecosystems on the continental scale. Proceedings of the National Academy of Sciences. 111 (26), 9549-9554 (2014).

2. Schäfer, R. B., Kühn, B., Malaj, E., König, A., \& Gergs, R. Contribution of organic toxicants to multiple stress in river ecosystems. Freshwater Biology. 61 (12), 2116-2128 (2016).

3. Andersen, M. E., \& Krewski, D. Toxicity testing in the 21st century: bringing the vision to life. Toxicological Sciences. 107 (2), $324-330$ (2008).

4. Rovida, C., \& Hartung, T. Re-evaluation of animal numbers and costs for in vivo tests to accomplish REACH legislation requirements for chemicals-a report by the transatlantic think tank for toxicology (t (4)). Altex. 26 (3), 187-208 (2009).

5. National Research Council. Toxicity testing in the 21st century: a vision and a strategy. National Academies Press (2007).

6. Mehta, G., Hsiao, A. Y., Ingram, M., Luker, G. D., \& Takayama, S. Opportunities and challenges for use of tumor spheroids as models to test drug delivery and efficacy. Journal of Controlled Release. 164 (2), 192-204 (2012).

7. Scholz, S., Fischer, S., Gündel, U., Küster, E., Luckenbach, T., \& Voelker, D. The zebrafish embryo model in environmental risk assessmentapplications beyond acute toxicity testing. Environmental Science and Pollution Research. 15 (5), 394-404 (2008).

8. Fraysse, B., Mons, R., \& Garric, J. Development of a zebrafish 4-day embryo-larval bioassay to assess toxicity of chemicals. Ecotoxicology and Environmental Safety. 63 (2), 253-267 (2006).

9. Noyes, P. D., Haggard, D. E., Gonnerman, G. D., \& Tanguay, R. L. Advanced morphological-behavioral test platform reveals neurodevelopmental defects in embryonic zebrafish exposed to comprehensive suite of halogenated and organophosphate flame retardants. Toxicological Sciences. 145 (1), 177-195 (2015).

10. Colón-Cruz, L., et al. Alterations of larval photo-dependent swimming responses (PDR): New endpoints for rapid and diagnostic screening of aquatic contamination. Ecotoxicology and Environmental Safety. 147, 670-680 (2018).

11. Kristofco, L.A., et al. Age matters: developmental stage of Danio rerio larvae influences photomotor response thresholds to diazinion or diphenhydramine. Aquatic Toxicology. 170 344-354 (2016).

12. Rihel, J., et al. Zebrafish behavioral profiling links drugs to biological targets and rest/wake regulation. Science. 327 (5963), $348-351$ (2010). 
13. Kokel, D., et al. Rapid behavior-based identification of neuroactive small molecules in the zebrafish. Nature Chemical Biology. 6 (3), $231-237$ (2010).

14. Bruton, T., Alboloushi, A., De La Garza, B., Kim, B.-O., \& Halden, R. U. in Contaminants of Emerging Concern in the Environment: Ecological and Human Health Considerations. 257-273 ACS Publications (2010).

15. Woudenberg, A.B., et al. Zebrafish embryotoxicity test for developmental (neuro) toxicity: Demo case of an integrated screening approach system using anti-epileptic drugs. Reproductive Toxicology. 49, 101-116 (2014).

16. Rodríguez-Gil, J., Cáceres, N., Dafouz, R., \& Valcárcel, Y. Caffeine and paraxanthine in aquatic systems: Global exposure distributions and probabilistic risk assessment. Science of the Total Environment. 612, 1058-1071 (2018).

17. Corrales, J., et al. Toward the Design of Less Hazardous Chemicals: Exploring Comparative Oxidative Stress in Two Common Animal Models. Chemical Research in Toxicology. 30 (4), 893-904 (2017).

18. Kimmel, C. B., Ballard, W. W., Kimmel, S. R., Ullmann, B., \& Schilling, T. F. Stages of embryonic development of the zebrafish. Developmental Dynamics. 203 (3), 253-310 (1995).

19. Kristofco, L. A., \& Brooks, B. W. Global scanning of antihistamines in the environment: Analysis of occurrence and hazards in aquatic systems. Science of the Total Environment. 592 477-487 (2017).

20. Saari, G. N., Scott, W. C., \& Brooks, B. W. Global assessment of calcium channel blockers in the environment: Review and analysis of occurrence, ecotoxicology and hazards in aquatic systems. Chemosphere. (2017).

21. Corrales, J., Kristofco, L.A., Steele, W.B., Yates, B.S., Breed, C.S., Williams, E.S., \& Brooks, B.W. Global Assessment of Bisphenol A in the Environment: Review and Analysis of Its Occurrence and Bioaccumulation. Dose-Response. 13 (3), 1559325815598308, (2015).

22. Berninger, J.P., et al. Effects of the antihistamine diphenhydramine on selected aquatic organisms. Environmental Toxicology and Chemistry. 30 (9), 2065-2072 (2011).

23. Brooks, B. W. Fish on Prozac (and Zoloft): ten years later. Aquatic Toxicology. 151, 61-67 (2014).

24. Huggett, D., Cook, J., Ericson, J., \& Williams, R. A theoretical model for utilizing mammalian pharmacology and safety data to prioritize potential impacts of human pharmaceuticals to fish. Human and Ecological Risk Assessment. 9 (7), 1789-1799 (2003).

25. Fitzsimmons, P. N., Fernandez, J. D., Hoffman, A. D., Butterworth, B. C., \& Nichols, J. W. Branchial elimination of superhydrophobic organic compounds by rainbow trout (Oncorhynchus mykiss). Aquatic Toxicology. 55 (1-2), 23-34 (2001).

26. Scheiner, S. M., \& Gurevitch, J. Design and Analysis of Ecological Experiments. Oxford University Press (2001).

27. Nakagawa, S. A farewell to Bonferroni: the problems of low statistical power and publication bias. Behavioral Ecology. 15 (6), $1044-1045$ (2004).

28. Bean, T.G., et al. Pharmaceuticals in water, fish and osprey nestlings in Delaware River and Bay. Environmental Pollution. 232 533-545 (2018).

29. Richendrfer, H., Pelkowski, S., Colwill, R., \& Creton, R. On the edge: pharmacological evidence for anxiety-related behavior in zebrafish larvae. Behavioural Brain Research. 228 (1), 99-106 (2012).

30. Padilla, S., Hunter, D., Padnos, B., Frady, S., \& MacPhail, R. Assessing locomotor activity in larval zebrafish: Influence of extrinsic and intrinsic variables. Neurotoxicology and Teratology. 33 (6), 624-630 (2011).

31. Sukardi, H., Chng, H. T., Chan, E. C. Y., Gong, Z., \& Lam, S. H. Zebrafish for drug toxicity screening: bridging the in vitro cell-based models and in vivo mammalian models. Expert Opinion on Drug Metabolism \& Toxicology. 7 (5), 579-589 (2011).

32. Ankley, G. T., \& Villeneuve, D. L. The fathead minnow in aquatic toxicology: past, present and future. Aquatic Toxicology. 78 (1), 91-102 (2006).

33. Hutson, L. D., \& Liang, J. O. Making an impact: zebrafish in education. Zebrafish. 6, 119+ (2009).

34. Hutson, L. D., Liang, J. O., Pickart, M. A., Pierret, C., \& Tomasciewicz, H. G. Making a difference: education at the 10th international conference on zebrafish development and genetics. Zebrafish. 9 (4), 151-154 (2012).

35. Kane, A., Salierno, J., \& Brewer, S. Fish models in behavioral toxicology: automated techniques, updates and perspectives. Methods in Aquatic Toxicology. 2, 559-590 (2005).

36. Rodriguez, A., et al. ToxTrac: a fast and robust software for tracking organisms. Methods in Ecology and Evolution. 9 (3), $460-464$ (2018).

37. Hamm, J., Wilson, B., \& Hinton, D. Increasing uptake and bioactivation with development positively modulate diazinon toxicity in early life stage medaka (Oryzias latipes). Toxicological Sciences. 61 (2), 304-313 (2001).

38. Kristofco, L. A., Haddad, S. P., Chambliss, C. K., \& Brooks, B. W. Differential uptake of and sensitivity to diphenhydramine in embryonic and larval zebrafish. Environmental Toxicology and Chemistry. 37, 1175-1181 (2018).

39. Steele, W. B., Kristofco, L. A., Corrales, J., Saari, G. N., Haddad, S. P,. Gallagher, E. P., Kavanagh, T. J., Kostal, J., Zimmerman, J. B., Voutchkova-Kostal, A., Anastas, P. T., \& Brooks, B. W. Comparative behavioral toxicology of two common larval fish models: exploring relationships between modes of action and locomotor responses. Science of the Total Environment. 460-461, 1587-1600 (2018). 